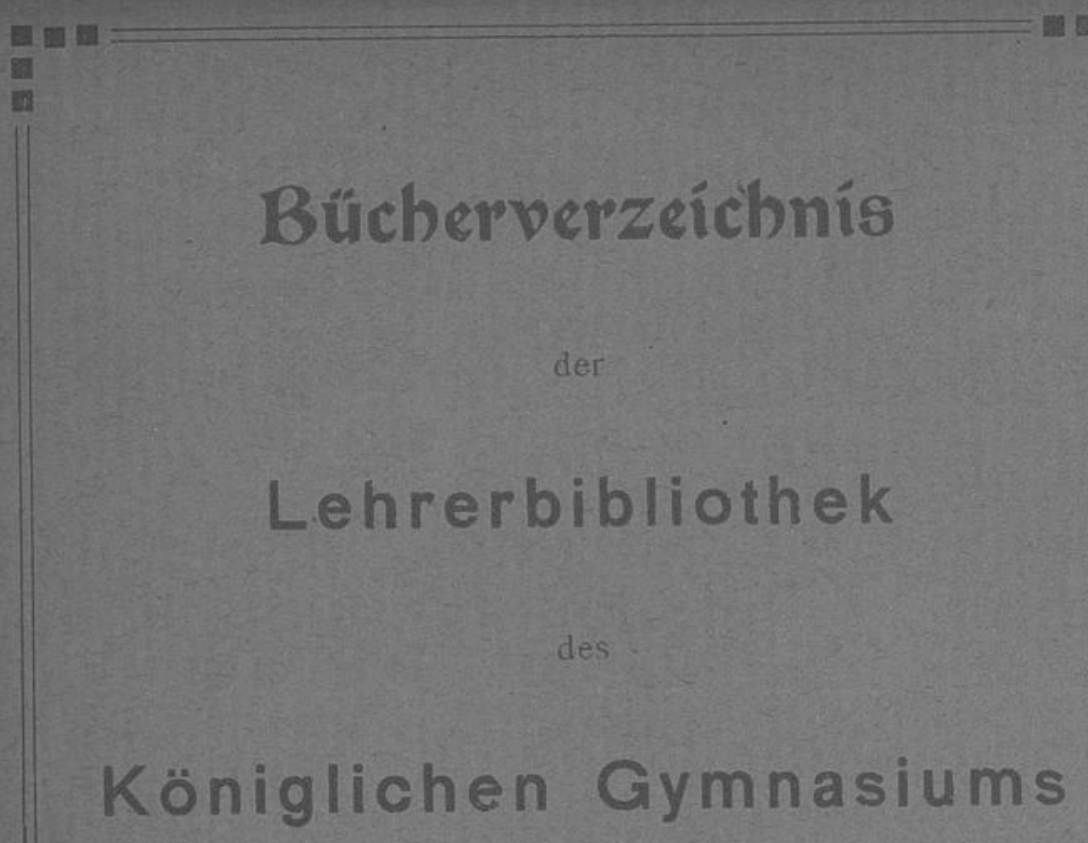

\title{
Königlichen Gymnasiums
}

\section{zu \\ Jauer}

von Professor Gotthard Heuber.

IV. Teil.

Griechische Schriftsteller.

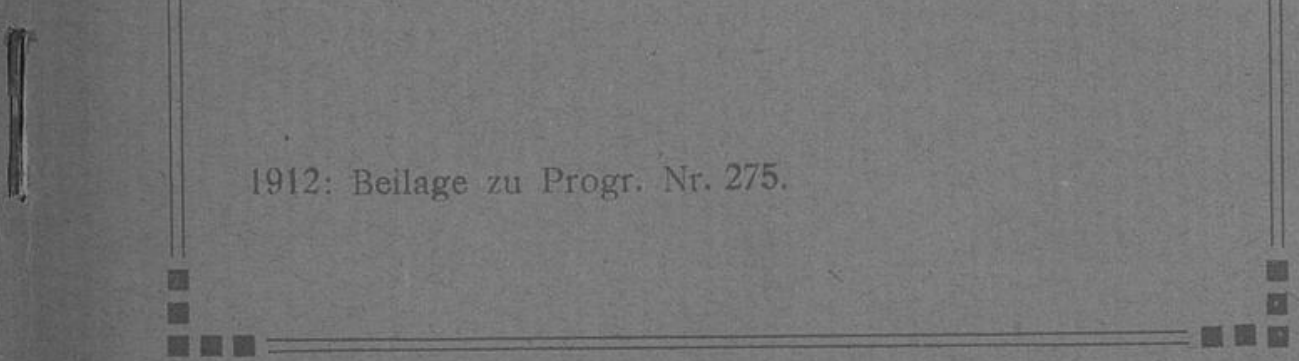




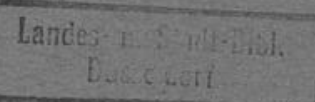

$$
44 . g \quad 304
$$




\title{
Bücherverzeichnis
}

\author{
der \\ Lehrerbibliothek \\ des \\ Königlichen Gymnasiums \\ Zu \\ Jauer \\ IV. Teil. \\ Griechische Schriftsteller.
}

1912: Beilage zu Progr. Nr. 275. 

Aeschines, orationes edidit Fridericus Franke. Leipzig: Teubner 1863.

- orationes edidit Ferdinandus Schultz. Leipzig: Teubner 1865.

- opera ad optimorum librorum fidem accurate edita. Editio stereotypa. Leipzig: Tauchnitz 1829.

- in Ctesiphontem oratio. rec. explicavit Andreas Weidner. Leipzig: Teubner 1872.

- Rede gegen Ktestiphon erklärt von A. Weidner. Berlin: Weidmann 1878.

A eschylus, tragoediae recensuit Godofredus Hermannus. Tom.I.II. Leipzig: Weidmann 1852.

- fabulae cum lectionibus et scholiis codicis Medicei et in Agamemnonem codicis Florentini ab Hieronymo Vitelli denuo collatis edidit $\mathrm{N}$. Wecklein.

Pars I. Textus. Scholia. Apparatus criticus. Berlin: Calvary \& Comp. 1885.

Pars. II. Appendix coniecturas virorum doctorum minus certas continens. Berlin: Calvary \& Comp. 1885.

- Choephorae ed. C. J. Blomfield. Leipzig: Hartmann 1824.

- Persae " ", " " , $\quad$ ", 1823.

- Prometheus vinctus ," " " " , , 1822.

- Agamemno ed. R. H. Klausen. edit. II. von R. Enger. Leipzig: Teubner 1863.

- Agamemnon erklärt von F. W. Schneidewin. 2. Aufl. von O. Hense. Berlin: Weidmann 1883.

- Orestie. Mit erklärenden Anmerkungen von N. Wecklein. Leipzig: Teubner 1888.

- Prometheus. Für den Schulgebrauch erklärt von N. Wecklein. Leipzig: Teubner 1872.

- Übersetzt von Joh. Gust. Droysen. 3. Aufl. Berlin: Hertz 1868.

- Lexikon Aeschyleum ed. Guilelmus Dindorfius. Leipzig: Teubner 1873 . 
Aesopus, Fabulae ed. Joh. Michael Heusinger; editio auctior et emendatior von Christ. Adolph Klotz. Eisenach und Leipzig: Wittekind 1776.

- Aesopi Phrygis vita et fabellae cum latina interpretatione.

- beigeb. Gabriae fabellae 43. Ex Aphthonii exercitamentis de fabula. de fabula ex imaginibus Philostrati graece et latine. Ex Hermogenis exercitamentis de fabula Prisciano interprete. Basel: Froben 1518.

Alexander Polyhistor, Commentatio de Alexandri Polyhistoris vita atque scriptis scripsit Josephus Rauch. Heidelberg: Mohr 1845.

Anaximenes, Ars rhetorica, quae vulgo fertur Aristotelis ad Alexandrum. rec. et illustr. Leonardus Spengel. Turici et Vitoduri 1844.

Andocides, orationes ed. Fridericus Blass. Leipzig: Teubner 1871. Anecdota Graeca, ed. Immanuel Becker. 3 Bde. Berlin: Nauck (Reimer) 1814-21.

- e codicibus manuscriptis bibliothec. Oxoniensium descr. J. A. Cramer. Vol. I-IV. Oxonii, e typographeo academico. $1835 / 41$.

- e codicibus manuscriptis bibliothecae regiae Parisiensis ed. J. A. Cramer. Vol. I-IV. Oxonii, e typographeo academico. $1835 / 41$.

- varia Graeca, musica, inetrica, grammatica ed. Guil. Studemund. Berlin: Weidmann 1886.

Anna Comnena, Alexias ex recensione Augusti Reifferscheidii. Vol. I.II. Leipzig: Teubner 1884.

Anthologia Graeca, carminum christianorum adornaverunt W. Christ et M. Paranikas. Leipzig: Teubner 1871.

Anthologia lyrica, sive lyricorum Graecorum veterum praeter Pindarum reliquiae potiores ed. Ed. Hiller. Leipzig: Teubner 1890.

Antoninus, Marcus, commentariorum, quos sibi ipsi scripsit libri XII. rec. Joannes Stich. Leipzig: Teubner 1882.

Appianus Alexandrinus, Romanarum historiarum quae supersunt graece et latine cum indicibus. Paris: Didot 1877.

Apollonius Dyscolus, Vol. I. fasc. I. Apollonii scripta minora aRichardoSchneidero edita continens. Leipzig:Teubner $18 \% 8$. 
Aratus, Phaenomena et diosemea. Cum annotatione critica ed. Philippus Buttmannus. Berlin: Mylius 1826.

Archimedes, Opera omnia cum commentariis Eutochii e codice Florentino recensuit, latine vertit notisque illustravit J. L. Heiberg. Vol. I-III. Leipzig: Teubner 1880/81. Aristides, ex recensione Guilielmi Dindorfii. 3 Vol. Leipzig: Weidmann 1829.

Aristides, Aelius Als Repräsentant der sophistischen Rhetorik des zweiten Jahrhunderts der Kaiserzeit von Dr. Hermann Baumgart. Leipzig: Teubner 1874.

Aristophanes, Comoediae ed. Theodorus Bergk. 2 Vol. Leipzig: Teubner 1857.

- Ausgewählte Komödien des Aristophanes erklärt von Theodor Kock.

Bd. 1. Die Wolken, 2. Aufl. Berlin: Weidmann 1862.

"2. Die Ritter, " " " 1853.

" 3. Die Frösche, " " 1856.

- Ausgewählte Komödien des Aristophanes erklärt von Theodor Kock.

Bd. 2. Die Ritter, 3 Aufl. Berlin: Weidmann 1884.

- Comoedia Plutus. recognovit... . Tiberius Hemsterhuis. editio nova appendice aucta. Leipzig: Fleischer 1811.

- Des Aristophanes Werke. Übersetzt von Joh. Gust. Droysen. 3. Aufl. 2 Teile in 1 Bd. Leipzig: Veit \& Comp. 1881.

- Die Lustspiele des Aristophanes. Übersetzt und erläutert von Hieronymus Müller. 3 Bde. Leipzig: Brockhaus 1861.

- De Aristophanis nubibus prioribus. Dissert. inaug. philol. scripsit Georg Schwandke. Halle: Karras 1898.

Aristoteles, Graece ex recensione Immanuelis Beckeri ed. Academia regia Borussica. 2 Bde. Berlin: Reimer 1831.

- Latine interpretibus variis ed. Academia regia Borussica. Berlin: Reimer 1831.

- Scholia in Aristotelem collegit Christianus Augustus Brandis ed. Academia regia Borussica. Berlin: Reimer 1836.

- opera omnia Graece et Latine cum indice nominum et rerum absolutissimo. 4 Bde. Paris: Didot 1862-67.

- Oeconomicus, Anonymi Oeconomica, Philodemi de vitiis et virtutibus ed. Carl Göttling. Jena: Walz 1830.

- Opera omnia Vol. I-XV. Leipzig: Tauchnitz 1830-32. 
Vol. I. Physica auscultatio. 1832.

II. Metaphysica. 1832.

III. De coelo libri IV, de generatione et corruptione libri II. 1831.

" IV. Meteorologica et de mundo liber. 1832

" V. Historia animalium. 1831.

"VI. Politicorum libri VIII et Oeconomica. 1831.

"VII. De anima, de sensu et sensili, de memoria, de somno, de somniis, de longitudine et brevitate vitae, de iuventute et senectute, de vita et morte, de respiratione. 1831.

"VIII. Organi pars I continens: Categorias, de interpretatione, topica, elenchos. 1832.

" IX. Organi pars II continens: Analytica priora et posteriora. 1832.

" X. Ethicorum ad Nicomachum libri X. 1831.

" XI. Physicorum libri VIII. 1831.

„ XII. De arte rhetorica libri III. 1831.

„XIII. De poetica liber et rhetorica ad Alexandrum 1831.

"XIV. Problemata. 1832. 2 Exemplare.

" XV. Moralia magna, moralia Eudemea et de virtutibus et vitiis libellus. 1832.

- Die Metaphysik des Aristoteles. Grundtext, Übersetzung und Commentar nebst erläuternden Abhandlungen von Dr. Albert Schwegler. 4 Bde. Tübingen: Fues 1847/48.

- Elementa logices Aristoteleae in usum scholarum ex Aristotele excerpsit convertit illustravit Fridericus Adolphus Trendelenburg. Editio V. Berlin: Bethge 1862.

- Dasselbe Editio VII. Berlin: Weber 1874.

- Erläuterungen zu den Elementen der aristotelischen Logik. Von Adolf Trendelenburg. Berlin: Bethge 1861.

- Ars rhetorica cum adnotatione Leonardi Spengel. Accedit vetusta translatio latina. 2 Vol. Leipzig: Teubner 1867.

- De arte poetica liber, iterum recensuit et adnotatione critica auxit Johannes Vahlen. Berlin: Vahlen 1874.

- Aristoteles' Poetik und Joh. Vahlens neueste Bearbeitung derselben von Leonhard Spengel. Leipzig: Teubner 1875. 
Aristoteles, Ethica Nicomachea edidit et commentario continuo instruxit G. Ramsauer. Adiecta est Franc. Susemihlii ad editorem epistota critica. Leipzig: Teubner 1878.

- Metaphysica recognovit W. Christ. Leipzig: Teubner 1886.

- A. qui ferebantur librorum fragmenta collegit Valentinus Rose. Leipzig: Teubner 1886.

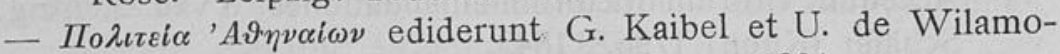
witz-Moellendorff. Berlin: Weidmann 1891.

- Grundzüge der verlorenen Abhandlung des Aristoteles über Wirkung der Tragödie von Jacob Bernays. Breslau: Trewendt 1857.

- Die Dialoge des Aristoteles in ihrem Verhältnis zu seinen übrigen Werken von Jacob Bernays. Berlin: Hertz 1863.

- Aristotelische Studien von Leonhard Spengel.

I. Nikomachische Ethik. München: Kgl. Akademie 1863.

II. Eudemische Ethik, Grosse Ethik, Politik. München: Kgl. Akademie 1865.

IV. Poetik. München: Kgl. Akademie 1866.

- Aristotelische Schriftstellen untersucht von Moritz Vermehren. 1. Heft. Zur Nikomachischen Ethik. Leipzig: Breitkopf \& Härtel 1864.

- Die verlorenen Schriften des Aristoteles von Emil Heitz. Leipzig: Teubner 1865.

- Aristoteles. Ein Abschnitt aus einer Geschichte der Wissenschaften nebst Analysen der naturwissenschaftlichen Schriften des Aristoteles. Von George Henry Lewes. Aus dem Englischen übersetzt von Julius Victor Carus. Leipzig: Brockhaus 1865.

- Der aristotelische Gottesbegriff mit Beziehung auf die christliche Gottesidee von L. F. Götz. Leipzig: Matthes 1870.

- Die Kunstlehre des Aristoteles. Ein Beitrag zur Geschichte der Philosophie von A. Döring. Jena: Dufft 1876.

- Die sogenannte Theologie des Aristoteles. Aus dem Arabischen übersetzt und mit Anmerkungen versehen von Fr. Dieterici. Leipzig: Hinrichs 1883.

- Die Staatslehre des Aristoteles. Ein Essay von A. C. Bradley. Autorisierte Übersetzung von F. Imelmann. Berlin: Gärtner 1886. 
Arrianus, Anabasis recognovit Carolus Abicht. Leipzig: Teubner 1903.

Athenaens, Dipnosophistarum libri XV. recensuit Georgius Kaibel. 3 Vol. Leipzig: Teubner 1887. 1890.

- Isaaci Casauboni animadversiones in Athenaei deipnosophistas. Accedunt virorum doctorum emendationes. 3 Vol. Leipzig: Schäfer (Kühn) 1796. 1843.

Babrios und die älteren Jambendichter. Griechisch mit metrischer Übersetzung und prüfenden und erklärenden Anmerkungen von J. A. Hartung. Leipzig: Engelmann 1858.

Bucolici Graeci, (Theocriti, Bionis et Moschi) carmina graece cum commentariis integris L. C. Valckenarii, Brunckii Ponpii ed. Heindorf. Vol. I und II. Berlin 1810.

Bionis et Moschi carmina ex codicibus Italis a se collatis edidit Christophorus Ziegler. Tübingen: Laupp 1868.

Callimachus, hymni et epigrammata recognovit Udalricus de Wilamowitz-Moellendorff. Berlin: Weidmann 1882.

- De C. Cydippa scripsit Carolus Dilthey. Leipzig: Teubner 1863.

- Callimachea edidit Otto Schneider. Vol. I. II. Leipzig: Teubner 1870/73.

Cleomedes, De motu circulari corporum caelestium libri duo. Ad novorum codicum fidem edidit et latina interpretatione instruxit Hermannus Ziegler. Leipzig: Teubner 1891.

Coluthns, Raptus Helenae recensuit Joannes Daniel a Lennep. editionem novam auctiorem cur avit Godofr. Henr. Schaefer. Leipzig: Hartmann 1825.

Comicorum Atticorum fragmenta edidit Theodorus Kock. $3 \mathrm{Vol}$. Leipzig: Teubner 1880. 1884. 1888.

Crates, De Cratete Mallota disputavit adiectis eius reliquiis Curtius Wachsmuth. Leipzig: Teubner 1860.

Demosthenes, Opera ad optimorum librorum fidem accurate edita. Editio stereotypa. Tom. I-V (3 Bde.) Leipzig: Tauchnitz 1829.

- orationes ex recensione Guilielmi Dindorfii. Vol. II. pars I. orationes XX-XXIII. Leipzig: Teubner 1862. 
Demosthenes, oratio de corona. ex recensione Imm. Beckeri passim mutata. explicuit Ludolphus Dissenius. Göttingen: Dieterich 1837.

- Die Kranzrede des D., das Meisterwerk der antiken Redekunst von Wilhelm Fox. Leipzig: Teubner 1880.

- oratio in Androtionem edidit Carolus Hermannus Funkhaenel. Leipzig: Weidmann 1832.

- Neun philippische Reden. Für den Schulgebrauch erklärt von C. Rehdantz. 2 Hefte (Heft 1 in 2. und 3. Aufl.) Leipzig: Teubner 1865 (66) und 1870.

- Ausgewählte Reden des D. Für den Schulgebrauch erklärt von J. Sörgel. 1. Bdchen. 5. Aufl. von A. Deuerling. Gotha: Perthes 1897.

- Neun philippische Reden. Textausgabe für den Schulgebrauch von Th. Thalheim. Leipzig: Teubner 1896.

- D'. oratio in Aristocratem edidit Guilielmus Weber. Jena: Cröker 1845.

- Ausgewählte Reden des D. erklärt von Anton Westermann. 1. Bdchen. 4. Aufl. Berlin: Weidmann 1860.

1. Bdchen. 8. Aufl. von J. Rosenberg. Berlin: Weidmann 1883.

Dio Cassins, Historia Romana. edit. primam curavit Ludovicus Dindorf, recognovit Joannes Melber. 2 Vol. Leipzig: Teubner 1890/94.

Diocles, Dioclis Carystii fragmenta quae supersunt. Dissertatio. Auctor Moritz Fraenkel. Berlin: Hayn 1840.

Diogenes Laertius, de vita et moribus philosphorum libri X. Leyden: Gryphius 1541.

- de vita, dogmatis et apophthegmatis clarorum philosophorum libri decem. ed. H. G. Huebner. 2 Vol. Leipzig: Köhler $1828 / 31$.

Dionysius Halicarnasensis, Antiquitatum Romanarum quae supersunt edidit Carolus Jacoby. Vol. I-III. Leipzig: Teubner 1885. 1888.1891.

Dionysius Halicarnaseus, Ars rhetorica recensuit Hermannus Usener. Leipzig: Teubner 1895.

- Werke. 3 Bde. Übersetzt von G. J. Schaller und A. H. Christian. Stuttgart: Metzler 1827-49. 
Dionysius Thrax, Ars grammatica edidit Gustav Uhlig. Leipzig: Teubner 1883.

Doxographi Graeci, collegit recensuit prolegomenis indicibusque instruxit Hermann Diels. Berlin: Reimer 1879.

Epicorum Graecorum fragmenta collegit disposuit. commentarium criticum adiecit Gottfried Kinkel. Vol. I. Leipzig: Teubner 1877.

- Parodorum ... Graecorum et Archestrati reliquiae. recognovit et enarravit P. Brandt. Leipzig: Teubner 1888.

Epicharmus, Leben und Schriften des Koers E. Nebst einer Fragmentensainmlung von Aug. O. Fr. Lorenz. Berlin: Weidmann 1864.

Epicteteae philosophiae monumenta edidit Joannes Schweighaeuser. Tom. I -V (6 Bde.) Leipzig: Weidmann $1799 / 1800$.

Epicurea edidit Hermannus Usener. Leipzig: Teubner 1887. Epigrammatum Graecorum Sylloge -, quae ante medium saeculum a. Chr. n. tertium incisa ad nos pervenerunt edidit Ernestus Hoftmann. Halle: Kämmerer 1893.

Epischen Poesie, Die Fragmente der - der Griechen bis zur Zeit Alexanders des Grossen, gesammelt von H. Düntzer. Köln: Eisen 1840.

Epistolographi Graeci, recensuit recognovit Rudolfus Hercher. Paris: Didot 1873.

Eratosthenes, carminum reliquiae disposuit et explicavit Eduardus Hiller. Leipzig: Teubner 1872.

- Die geographischen Fragmente des Eratosthenes, neu gesammelt, geordnet und besprochen von Hugo Berger. Leipzig: Teubner 1880.

Enelides, Litterargeschichtliche Studien über E. von J. L. Heiberg. Leipzig: Teubner 1882.

Euripides, Bacchae in usum studiosae iuventutis recensuit et illustravit Petrus Elmsley. Leipzig: Hartmann 1822.

- Electra ed. Seidler. (Titelblatt fehlt.)

- Hecuba denuo recensuit Godofredus Hermannus. Leipzig: Weidmann 1831.

- Hercules Furens recensuit Godofredus Hermannus. Leipzig: Fleischer 1810. 
- Heracles. Erklärt von Ulrich von Wilamowitz-Moellendorff. Bd. 1. Einleitung in die attische Tragödie. Berlin: Weidmann 1889.

Bd. 2. Text und Commentar. Berlin: Weidmann 1889.

- Hippolytus. Griechisch und deutsch von Ulrich von Wilamowitz-Moellendorff. Berlin: Weidmann 1891.

- Jon recensuit et commentario instruxit Henricus van Herwerden. Utrecht: Kemink \& Sohn 1875.

- Iphigenie auf Taurien zum Schulgebrauche mit erklärenden Anmerkungen versehen von Wolfg. Bauer. München: Lindauer 1872.

- Ausgewählte Tragödien erklärt von F. G. Schöne. 2. Bdchen.: Iphigenia in Taurien. 3. Aufl. von H. Köchly. Berlin: Weidmann 1872.

- Ausgewählte Tragödien. Für den Schulgebrauch erklärt von N. Wecklein.
1. Bdchen.
3. "Bacchen. ", " 1879

- Sämtliche Tragödien des E. Metrisch übertragen von Franz Fritze, vollendet von Theodor Kock. 4 Bde. Berlin: Schindler 1857. 1859. 1869.

- Diatribe in Euriqidis perditorum dramatum reliquias edidit Ludw. Casp. Valckenarius. Leipzig: Hartmann 1824.

Eusebius, Chronicorum liber prior edidit Alfred Schoene. Berlin: Weidmann 1875.

Excerpten-Litteratar, Beiträge zur griechischen - von Arthur Kopp. Berlin: Gärtner 1887.

Galenus, Claudius, Scripta minora recensuerunt Jo. Marquardt, Iw. Mueller, Ge. Helmreich. 3 Vol. Leipzig: Teubner 1884. 1891. 1893.

Heraclitus, Die Heraklitischen Briefe. Ein Beitrag zur philosoph. und religionsgesch. Litteratur von Jacob Bernays. Berlin: Hertz 1869.

Hermippus, H. Smyrnaei Peripatetici fragmenta edidit Adalbertus Lozynski. Bonn: Habicht 1832.

Herodianus, ab excessu Divi Marci libri octo edidit Ludovicus Mendelssohn. Leipzig: Teubner 1883. 
- Herodiani technici reliquiae collegit, disposuit, emendavit, explicavit, praefatus est Augustus Lentz. 3 Tom. Leipzig: Teubner 1867. 1870.

- scripta tria emendatiora edidit K. Lehrs. Königsberg: Samter 1848.

Herodotus, Herodoti Halicarnassensis Musae ed. J. C. F. Baehr. ed. II. 4 Vol. Leipzig: Hahn 1856-61.

- Herodoti historiarum libri IX ed. H. R. Dietsch. ed. II curavit H. Kallenberg. 2 Vol. Leipzig: Teubner 1898/99.

- Herodoti historiae. Ad recensionem suam recognovit Henricus Stein. 2 Vol. Berlin: Weidmann 1884.

- erklärt von Heinrich Stein. 5 Bde. 2. bezw. 3. und 4. Aufl. Berlin: Weidmann 1856-82.

- Für den Schulgebrauch erklärt von Dr. K. Abicht. Bd. 3, 4, 5 . Leipzig: Teubner 1873/74.

H.'s zweites Buch mit sachlichen Erläuterungen herausgegeben von Alfred Wiedemann. Leipzig: Teubner 1890.

Lexicon Herodoteum ed. Joh. Schweighaeuser. Adiecta est appendix tractatus quosdam complectens de dialecto Jonica. London: Priestley 1830.

Die Geschichten des Herodot. Deutsch von Heinrich Stein. 2 Bde. Oldenburg: Schmidt 1875.

Die Entstehung des Herodotischen Geschichtswerkes. Eine kritische Untersuchung von Adolf Bauer. Wiẹn: Braumüller 1878.

Quaestionum criticarum de dialecto Herodotea libri quattuor scripsit F. J. C. Bredow. Leipzig: Teubner 1846.

Herondas, Mimiamboi a first recension by W. G. Rutherford. London: Macmillan \& Co. 1891.

Hesiodus, Carmina rec, et commentariis instruxit Carolus Goettlingius. Ed. III. quam curavit Joh. Flach. Leipzig: Teubner 1878.

Hesiodi Ascraei opera, quae quidem exstant, omnia graece cum interpretatione Latina .... ed. J. F. Possel. Leipzig: Lamberg 1603.

Die Werke und Tage des H. Nach ihrer Composition geprüft und erklärt von A. Steitz. Leipzig: Teubner 1869. 
Hesiodi quod fertur scutum Herculis ex recognitione et cum animadversionibus Fr. A. Wolfii ed. C. F. Ranke. Quedlinburg und Leipzig: Bass 1840.

Glossen und Scholien zur Hesiodischen Theogonie mit Prolegomena. Herausgegeben von H. Flach. Leipzig: Teubner 1876.

Das System der Hesiodischen Kosmogonie dargestellt von H. Flach. Leipzig: Teubner 1874.

Hesychius, Hesychii Milesii onomatologi, quae supersunt. cum prolegomenis edidit Joannes Flach. Leipzig: Teubner 1882.

- Hesychii Alexandrini Lexicon post Joannem Albertum recensuit Mauritius Schmidt. 5 Vol. (in 3 Bden.) Jena: Mauk $1858-68$.

Hipparchus, Die geographischen Fragmente des H. zusammengestellt und besprochen von Hugo Berger. Leipzig: Teubner 1869.

Hippocrates, Opera omnia quae exstant Latina interpretatione et annotationibus illustrata ed. Anutius Foesius. Frankfurt: Wechel 1595.

- Hippocratis Coi de aëre aquis et locis liber denuo recensitus et varietate lectionis Foesiana et Coraiana instr. Ch. Petersen. Hamburg: Perthes \& Besser 1833.

Historicorum Graecorum fragmenta ed. et illustr. Car. et Theod. Müller. 5 Vol. Paris: Didot 1848-73.

Historici Graeci minores ed. L. Dindorf. 2 Vol. Leipzig: Teubner 1870/71.

Homerus, Batrachomyomachia, hoc est ranarum et murium pugna. Graece et latine. Basel: Froben 1518.

Ilias. Für den Schulgebrauch erklärt von K. Fr. Ameis. 4. bezw. 3. Aufl. von C. Hentze. 4 Teile. Leipzig: Teubner $1882-86$.

Odyssee. Für den Schulgebrauch erklärt von K. Fr. Ameis. 8. bezw. 7. und 6. Aufl. von C. Hentze. 2 Bde. Leipzig: Teubner $1880-84$.

Ilias ad fidem librorum optimorum ed. J. La Roche. 2 Bde. Leipzig: Teubner $1873-76$.

Odyssea ad fidem librorum optimorum ed. J. La Roche.

2 Bde. Leipzig: Teubner 1867/68. 
Iliadis carmina seiuncta discreta emendata prolegomenis et apparatu critico instructa ed. Guil. Christ. 2 Vol. Leipzig: Teubner 1884/85.

Ilias seu potius omnia eius quae exstant opera. Studio et cura O. B. Giphanii quam emendatissime edita cum eiusdem scholiis et indicibus novis. Strassburg: Rihel 1572.

Aristarchs Homerische Textkritik nach den Fragmenten des Didymos dargestellt und beurteilt von Arthur Ludwich. 2 Teile. Leipzig: Teubner 1884/85.

De Aristarchi studiis Homericis scripsit K. Lehrs. Leipzig: Hirzel 1865.

Scholia Graeca in Homeri Iliadem ex codicibus aucta et emendata ed. Guil. Dindorf. 4 Tomi. Oxford: Clarendon $1875-77$.

Scholia Graeca in Homeri Odysseam ex codicibus aucta et emendata ed. Guil. Dindorf. 2 Tomi. Oxford: Akad. Buchdruckerei 1855 .

Eustathii commentarii ad Homeri Iliadem. Ad fidem exempli Romani editi. 4 Tomi. Leipzig: Weigel 1827-30.

Eustathii commentarii ad Homeri Odysseam. Ad fidem exempli Romani editi. 2 Tomi. Leipzig: Weigel 1825/26.

Index in Eustathii commentarios in Homeri Iliadem et Odysseam. Studio Matthaei Devarii. Ad fidem exempli Romani. Leipzig: Weigel 1828.

Ameis-Hentze, Anhang zu Homers Ilias Schulausgabe. 8 Hefte. Leipzig: Teubner 1882-1900.

Apollonii Sophistae lexicon Homericum ex recensione Immanuelis Beckeri. Berlin: Reimer 1833.

Doederlein, Ludwig, Homerisches Glossarium. 3 Bde. Erlangen: Enke $1850-58$.

Ebeling, H., Lexicon Homericum. 2 Vol. Leipzig: Teubner 1880/85.

Goebel, Anton, Lexilogus zu Homer und den Homeriden. 2 Bde. Berlin: Weidmann 1878/80.

Adam, Ludwig, Die Odyssee und der epische Cyklus. Wiesbaden: Niedner 1880.

Baer, Karl Ernst von, Über die Homerischen Lokalitäten in der Odyssee. Braunschweig: Vieweg \& Sohn 1878. 
Blackwell, Untersuchung über Homers Leben und Schriften. Aus dem Englischen übersetzt von Johann Heinrich Voss. Leipzig: Weygand 1776.

Blass, Friedrich, Die Interpolationen in der Odyssee. Halle: Niemeyer 1904.

Bonitz, Hermann, Über den Ursprung der Homerischen Gedichte. Vortrag. 5. Aufl. bes. von R. Neubauer. Wien: Gerold 1881.

Cauer, Paul, Grundfragen der Homerkritik. Leipzig: Hirzel 1895.

Classen, Joh., Beobachtungen über den homerischen Sprachgebrauch. Frankfurt a. M.: Winter 1867.

Drerup, Engelbert, Homer. München: Kirchheim 1903. Düntzer, Heinrich, Die homerischen Fragen. Leipzig: Hahn 1874.

Friedrich, J. B., Die Realien in der Iliade und Odyssee. Erlangen: Enke 1856.

Hartel, Wilhelm, Homerische Studien. Beiträge zur Homerischen Prosodie und Metrik. Berlin: Vahlen 1873.

Helbig, W., Das Homerische Epos aus den Denkmälern erläutert. Archäologische Untersuchungen. Leipzig:Teubner 1884.

Hennings, P. D. Ch., Homers Odyssee. Ein kritischer Kommentar. Berlin: Weidmann 1903.

Hercher, Rudolf, Homerische Aufsätze. Berlin: Weidmann 1881.

Hinrichs, Gustav, Herr Dr. Karl Sittl und die homerischen Äolismen. Berlin: Weidmann 1884.

Jäger, Oskar, Homer und Horaz im Gymnasial-Unterricht. München: Beck 1905.

Jebb, R. C., Homer. Eine Einführung in die Ilias und Odyssee. Übersetzung von E. Schlesinger. Berlin: Calvary \& Co. 1893.

Kammer, Eduard, Ein ästhetischer Kommentar zu Homers Ilias. Paderborn: Schöningh 1889.

- Kritisch-ästhetische Untersuchungen betreffend die Gesänge $M N \Xi O$ der Ilias. Königsberg: Hartung 1887. 
- Die Einheit der Odyssee nach Widerlegung der Ansichten von Lachmann-Steinthal, Koechly, Hennings und Kirchhoff. Anhang: Homerische Blätter von Prof. Dr. Lehrs. Leipzig: Teubner 1873.

Kayser, R. L., Homerische Abhandlungen herausgegeben von Hermann Usener. Leipzig: Teubner 1881.

Kirchhoff, A., Die Composition der Odyssee. Gesammelte Aufsätze. Berlin: Hertz 1869.

Koës, G. H. C., Commentatio de discrepantiis quibusdam in Odyssea occurrentibus. Hafniae: Seidelin 1806.

Lehrs, K., Quaestiones epicae. Königsberg: Bornträger 1837.

Leeuwen, J. van und Mendes da Costa M. B., Der Dialekt der Homerischen Gedichte. Aus dem Holländischen übersetzt von Dr. E. Mehler. Leipzig: Teubner 1886.

Naegelsbach, Carl Friedrich, Anmerkungen zur Ilias. Nebst einigen Exkursen. 3. Aufl. bearbeitet von Dr. Georg Autenrieth. Nürnberg: Geiger 1864.

Niese, Benedikt, Die Entwicklung der Homerischen Poesie. Berlin: Weidmann 1882.

Nitzsch, Gregor Wilhelm, Erklärende Anmerkungen zu Homers Odyssee. 3 Bde. Hannover: Hahn 1826 - 40. * 1901.

Reichel, Wolfgang, Homerische Waffen. Wien: Hölder

Rothe, Carl, Die Ilias als Dichtung. Paderborn: Schöningh 1910.

Schrader, Hermann, Porphyrii quaestionum Homericarum ad Iliadem pertinentium reliquiae. Fasc. I. Leipzig: Teubner 1880.

- Porphyrii quaestionum Homericarum ad Odysseam pertinentium reliquiae. Leipzig: Teubner 1890.

Schreiner, Joseph, Homers Odyssee - ein mysteriöses Epos. Braunschweig und Leipzig: Sattler 1901.

Sitzler, Jakob, Ein ästhetischer Kommentar zu Homers Odyssee. Paderborn: Schöningh 1902.

Spohn, Wilhelm, Dissertationis de extrema Odysseae parte inde a rhapsodiae $\psi$ versu ccxcvII aevo recentiore orta quam Homerico pars prior. Leipzig: Teubner 1815. 
Terpstra, J., Antiquitas Homerica. Leiden: Luchtmans 1831.

Volkmann, Richard, Geschichte und Kritik der Wolfschen Prologomena zu Homer. Leipzig: Teubner 1874.

Wolf, Friedrich August, Prolegomena ad Homerum. Editio nova cum notis ineditis Immanuelis Beckeri. Berlin: Calvary \& Co. 1872.

Gehring, A, Index Homericus. Leipzig: 1891.

Homerischen Hymnen, Die, Herausgegeben und erläutert von Dr. Albert Gemoll. Leipzig: Teubner 1886.

Homeri Hymni et Epigrammata ed. Godofredus Hermannus. Leipzig: Weidmann 1806.

Homeri Hymni et Batrachomyomachia denuo recensuit... Augustus Matthiae. Leipzig: Weidmann 1805.

Hymnus Cereris Homericus ed. Franz Bücheler. Adiectum est manuscripti simulacrum. Leipzig: Teubner 1869.

Homeri Hymnus in Cererem, nunc primum editus a Davide Ruhnkenio. Leipzig: Lehnhold 1849.

Animadversiones in Hymnos Homericos cum Prolegomenis de cuiusque consilio, partibus, aetate; auctore Aug. Matthiae. Leipzig: Weidmann 1800.

Kritische Blätter zu den homerischen Hymnen und zu Xenophons Anabasis. Striegau: Tschörner 1890.

epische Cyklus, der, oder die Homerischen Dichter von F. G. Welcker. Bonn: Weber 1865 .

Hyperides, orationes quattuor cum ceterarum fragmentis ed. F. Blass. Leipzig: Teubner 1881.

Jamblichus, de communi mathematica scientia liber ad fidem codicis Florentini ed. Nicolaus Festa. Leipzig: Teubner 1891.

- Protrepticus. Ad fidem codicis Florentini ed. Hermenegildus Pistelli. Leipzig: Teubner 1888.

Inseriptionum, Sylloge - Graecarum ed. Guilelmus Dittenberger. Fasc. I. II. Leipzig: Hirzel 1883.

Josephi, Flavii, opera omnia. Post Immanuelem Beckerum recogn. S. A. Naber. 3 Vol. Leipzig: Teubner 1888 -92.

- Die jüdischen Altertümer übersetzt und mit Anmerkungen versehen von Dr. K. Martin. 2 Bde. Köln: Bachem $1852 / 53$. 
Isaeus, orationes XI cum aliquot deperditarum fragmentis recognovit et commentarios adiecit G. F. Schoemann. Greifswald: Moritz 1831.

- orationes cum deperditarum fragmentis post Carolum Scheibe iterum edidit Th. Thalheim. Leipzig: Teubner 1903.

Isocrates, orationes recognovit praefatus est indicem nominum addidit Gustavus Eduardus Benseler. Editio altera stereotypa curante Friderico Blass. Vol. I. II. Leipzig: Teubner $1898 / 1907$.

- Ausgewählte Reden. Für den Schulgebrauch erklärt von Dr. Otto Schneider.

1. Bdchen. 2. Aufl. Leipzig: Teubner 1874.

2. " 3. " besorgt. von Dr. M. Schneider. Leipzig: Teubner 1886.

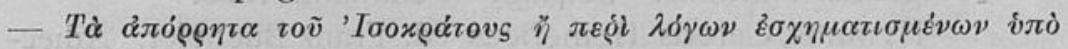
'A Kvлюtavoṽ. Athen: Hermes 1871.

Quaestiones Isocrateae. Dissertatio inauguralis scripsit Carolus Muenscher. Göttingen: Dieterich 1895.

Julianus, Juliani Imperatoris quae supersunt praeter reliquias apud Cyrillum omnia. recensuit Fridericus Carolus Hertlein. 2 Vol. Leipzig: Teubner 1875/76.

Juliani Imperatoris librorum contra Christianos quae supersunt. Collegit recensuit prolegomenis instruxit Carolus Joannes Neumann. Insunt Cyrilli Alexandri fragmenta Syriaca ab Eberhardo Nestle edita. Leipzig: Teubner 1880.

Libanius, Libanii Sophistae epistolae edidit, latine convertit et notis illustravit Joannes Christophorus Wolfius. Amsterdam: Jansson-Wäsberg 1738.

Lneianus, Luciani Samosatensis opera cum nova versione, Graecis scholiis ac notis ed. Tib. Hemsterhusius et Jo. Fred. Reitzius. 3 Bde. Amsterdam: Wetstein 1743 - 46.

- Index verborum ac phrasium Luciani, sive lexicon Lucianeum, ad editiones omnes, maxime novissimam Wetstenianam, concinnatum a Car. Conr. Reitzio. Utrecht: Besseling: 1746.

- Recognovit Julius Sommerbrodt. 4 Vol. Berlin: Weidmann $1886-96$. 
- Luciani Alexander, Demonax, Gallus, Icaromenippus, Philopseudes, ad Hesiodum, navigium. Ex conformatione Fr. Volckmari Fritzsche. Praecedunt quaestiones Lucianeae. Leipzig: Hartmann 1826.

- Ausgewählte Schriften des Lucian erklärt von Julius Sommerbrodt.

1. Bdchen.: Über Lucians Leben und Schriften. Lucians Traum. Charon. Timon.

2. Bdchen.: Nigrinus. Der Hahn. Icaromenipus.

3.

Wie man Geschichte schreiben soll. Die Rednerschule. Der Fischer. Der ungebildete Büchernarr. Über die Pantomimik. Berlin: Weidmann 1860. 1853.1857.

- 1. Bdchen. Über Lucians Leben und Schriften. Lucians Traum. Charon. Timon. Berlin: Weidmann 1872.

- Lucian und die Kyniker von Jacob Bernays. Mit einer Übersetzung der Schrift Lucians „Über das Lebensende des Peregrinus." Berlin: Hertz 1879.

Lycophron, Alexandra ed. Ludovicus Bachmannus. Vol. I. Leipzig: Hinrichs 1830.

- Alexandra recensuit scholia vetera codicis Marciani addidit Godofredus Kinkel. Leipzig: Teubner 1880.

- Tzetzae Scholia ad Lycophronem ed. Christ. Gottfried Müller. 3 Vol. Leipzig: Vogel 1811.

Lycurgus, oratio in Leocratem ed. Fr. Blass. Leipzig: Teubner 1899.

- Rede gegen Leocrates erklärt von Adolph Nicolai. Berlin: Weidmann 1875.

Lyriei, Poetae lyrici Graeci rec. Theodorus Bergk. 3 Vol. Leipzig: Teubner 1878-82.

Lyriker, Anthologie aus den Lyrikern der Griechen. Für den Schul- und Privatgebrauch erklärt von E. Buchholz.

1. Bdchen.: Die Elegiker und Jambographen. Leipzig: Teubner 1886 .

Lysias, orationes . . recensuit Carolus Scheibe. editio altera. Leipzig: Teubner 1900.

- orationes ... recensuit Theodorus Thalheim. editio maior. Leipzig: Teubner 1901. 
- Ausgewählte Reden. Für den Schulgebrauch erklärt von Hermann Frohberger. Kleinere Ausgabe. Leipzig: Teubner 1875.

- Ausgewählte Reden. Für den Schulgebrauch bearbeitet von Th. Thalheim. 2 Bde. Leipzig: Teubner 1895/92.

- Ausgewählte Reden erklärt von Dr. R. Rauchenstein. Berlin: Weidmann 1872.

- Ausgewählte Reden erklärt von Dr. R. Rauchenstein. 2. Bdchen besorgt von Karl Fuhr. Berlin: Weidmann 1881.

Mnaseas, Mnaseae Patarensis fragmenta. Particula prior. Dissertatio philologica. scripsit Eugen Mehler. Bonn: Krüger 1846.

Oppianus, Oppiani poetae Cilicis, de venatione libri IV, de piscatu libri $\mathrm{V}$, cum interpretatione Latina, commentariis et indice rerum ... ed. Conrad Rittershusius. Leyden: Plantin 1597.

Oratores Attici, ex recensione Immanuelis Beckeri. 5 tomi. Berlin: Reimer 1823/24.

Panaetius, Disputatio historico-critica de Panaetio Rhodio philosopho Stoico prop. J. G. van Lynden. Leyden: Honkoop 1802.

Paroemiographi Graeci ediderunt E. L. a. Leutsch et F. G. Schneidewin. Göttingen: Vandenhoeck \& Ruprecht 1839.

Pausanias, Descriptio arcis Athenarum in usum scholarum edidit Otto Jahn. Bonn: Mark 1880.

Philetas, Philetae Coi, Hermesianactis Colophonii atque Phanoclis reliquiae disposuit, emendavit, illustravit Nicolaus Bachius. Accedunt D. Lennepii et D. Ruhnkenii observationes integrae. Halle: Gebauer 1829.

Philodemus, de musica librorum quae exstant edidit Joannnes Kemke. Leipzig: Teubner 1884.

Philosophi, Philosophorum Graecorum fragmenta recensuit ... indicibus instruxit Fr. Guil. Aug. Mullachius. 2 Vol. Paris: Didot 1860/67.

Philostratus, Flavii Philostrati opera ed. C. L. Kayser. 2 Vol. Leipzig: Teubner 1870/71.

Phrynichus, Phrynichi eclogae nominum et verborum Atticorum ed. Chr. A. Lobeck. Accedunt fragmentum Herodiani et 
notae, praefationes Nunnesii et Pauwii et parerga . . . Leipzig: Weidmann 1820.

Pindarus, Pindari opera quae supersunt ed. Augustus Boeckhius. 2 Vol. Leipzig: Weigel $1811 / 19$.

- Pindari carmina quae supersunt cum deperditorum fragmentis selectis ed. Ludolphus Dissenius ed. II curavit F. G. Schneidewin. 2 Vol. Gotha: Hennings 1843/47.

- Lexicon Pindaricum composuit Joannes Rumpel. Leipzig: Teubner 1883.

- Pindars Leben, Weltanschauung und Kunst dargestellt von G. Bippart. Jena: Hochhausen 1848.

- Die Pindarscholien. Eine kritische Untersuchung zur philologischen Quellenkunde von K. Lehrs. Leipzig: Hirzel 1873.

- Pindars Siegeslieder erklärt von Friedrich Mezger. Leipzig: Teubner 1880.

- Pindars Leben und Dichtung dargestellt von Leopold Schmidt. Bonn: Marcus 1862.

- Pindars Olympische Siegesgesänge. Griechisch und deutsch von Moritz Schmidt. 1. Bd. Jena: Mauke 1869.

Plato, Piatonis quae exstant opera. Accedunt Platonis quae feruntur scripta rec., in linguam Latinam convertit, annotationibus explanavit indicesque adiecit Fridericus Astius. 11 tomi (10 Teile). Leipzig: Weidmann 1819 - 32 .

Platonis dialogi secundum Thrasylli tetralogias dispositi. ex recensione Caroli Friderici Hermanni. Vol. I-VI. Leipzig: Teubner 1849 -53.

Platonis dialogi secundum Trasylli tetralogias dispositi. Post C. F. Hermannum recognovit Martinus Wohlrab. Vol. I. Leipzig: Teubner 1887.

Platons Verteidigungsrede des Sokrates und Kriton. Für den Schulgebrauch erklärt von Heinrich Bertram. Gotha: Perthes 1894.

Platons Ausgewählte Schriften. Für den Schulgebrauch erklärt von Christian Cron und Julius Deuschle. T. 1-4 (Apologie, Kriton, Gorgias, Laches, Protagoras.) T. 1 (2. Aufl.) Leipzig: Teubner 1859-61.

Platons Aúsgewählte Schriften. Für den Schulgebrauch erklärt von Christian Cron und Julius Deuschle. T. 5 
(Symposion) erklärt von Arnold Hug. Leipzig: Teubner 1876.

Platonis Symposium in usum studiosae iuventutis et scholarum cum commentario critico edidit Georg. Ferd. Rettig. Halle: Waisenhaus 1875.

Platonis Protagoras recognovit et cum G. Stallbaumii suisque annotationibus edidit J. S. Kroschel. Leipzig: Teubner 1865.

Platonis dialogos selectos recensuit et commentariis in usum scholarum instruxit Godofredus Stallbaum.

Vol I. Sect. II. continens Phaedonem

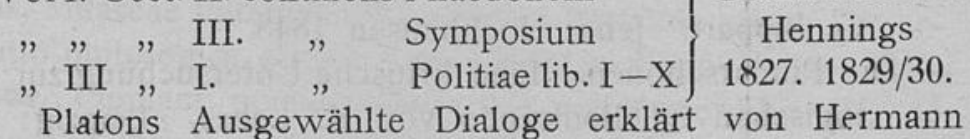
Sauppe.

2. Bdchen. Protagoras. Berlin: Weidmann 1857.

Platons Ausgewählte Dialoge erklärt von K. Schmelzer. Phädrus. Berlin: Weidmann 1882.

Platens Ausgewählte Dialoge erklärt von K. Schmelzer. 6. Bd. Menon, Euthyphron. Berlin: Weidmann 1883.

Politia, recensuit, prolegomenis et commentariis illustravit Godofredus Stallbaum. 2 Vol. Gotha und Erfurt: Hennings 1858/59.

Platons Euthyphron. Für den Schulgebrauch erklärt von Martin Wohlrab. Leipzig: Teubner 1873.

Platons Phaedo explanatus et emendatus prolegomenis et annotatione Danielis Wyttenbachii. Leipzig: Hartmann 1825.

Platons Werke. Griechisch und deutsch mit kritischen und erklärenden Anmerkungen. Teil VI, XV, XVI. Phädrus, Timäus, Kritias, Parmenides. (1 Bd.) Leipzig: 1846. 1853.1854.

Platonicarum, Timaei Sophistae lexicon vocum - ed. David Ruhnkenius. Leyden: Luchtmans 1789.

Platons sämtliche Werke übersetzt von Hieronymus Müller, mit Einleitungen begleitet von Karl Steinhart. 8 Bde. Leipzig: Brockhaus 1850-66.

Das Christliche im Plato und in der platonischen Philosophie, entwickelt und hervorgehoben von C. Ackermann. Hamburg: Perthes 1835. 
Die Frage über Geist und Ordnung der Platonischen Schriften beleuchtet aus Aristoteles von Eduard Alberti. Leipzig: Teubner 1864.

Platons Phädon, philosophisch erklärt und durch die späteren Beweise für die Unsterblichkeit ergänzt von J. Baumann. Gotha: Perthes 1889 .

Über die Aufgabe des platonischen Dialogs: Kratylos. von Theodor Benfey. Göttingen: Dieterich 1866.

Platonische Studien von H. Bonitz. Berlin: Vahlen 1886.

Die platonischen Mythen, inbesondere der Mythos im platonischen Phädrus. von Julius Deuschle. Hanau: König 1854.

Die platonische Sprachphilosophie. Dargestellt von Julius Deuschle. Marburg: Elwert 1852.

Initia philosophiae Platonicae. auctore Phil. Guil. van Heusde. 2 Vol. $(1$ Bd.) Utrecht: Altheer 1827-31.

Zur Erkenntnis der geistigen Entwicklung und der schriftstellerischen Motive Platos. Eine Studie von Karl Joel. Berlin: Gärtner 1887.

Platon und die Rhetorik. Eine philosophische Studie von J. V. Novák. Leipzig: Teubner 1883.

Platons philosophische Entwicklung von Hans Raeder. Leipzig: Teubner 1905.

Sieben Bücher zur Geschichte des Platonismus von Heinrich von Stein. 3 Teile. Göttingen: Vandenhoeck \& Ruprecht $1862-75$.

Die genetische Entwicklung der platonischen Philosophie von Franz Susemihl. 2 Teile, Leipzig: Teubner 1855-60.

Die Platonische Frage. Eine Streitschrift gegen Zeller. von Gustav Teichmüller. Gotha: Perthes 1876.

Platonis de ideis et numeris doctrina ex Aristotele illustrata scripsit Fr. Ad. Trendelenburg. Leipzig: Voge I 1826.

Platon. Von Wilhelm Windelband. Stuttgart: Frommann 1905.

Plotinus, Enneades praemisso Porphyrii de vita Plotini deque ordine librorum eius libello edidit Ricardus Volkmann. 2 Vol. Leipzig: Teubner 1883/84. 
Quaestionum de dialectica Plotini ratione fasc. 1. scripsit C. H. A. Steinhart. Naumburg: Klaffenbach 1829.

Plntarchus, Pl. Chaeronensis quae supersunt omnia ed. Joh. Georg Hutten. 14 Vol. Tübingen: Cotta 1791-1804.

- Moralia recognovit Gregorius N. Bernardakis. 5 Vol. Leipzig: Teubner 1888-93.

- Moralia graeca emendavit ..., latinam Xylandri interpretationem castigatam subiunxit, animadversiones ...., indices . adiecit Daniel Wyttenbach. 8 Tomi (15 Bde.) Oxford: Clarendon 1795-1830.

- Vitae parallelae ad optimorum librorum fidem edidit G. H. Schaefer. 9 Tomi. Leipzig: Tauchnitz 1812-14.

- Vitae parallelae ex recensione Caroli Sintenis. 4 Vol. Leipzig: Koehler 1839-46.

- Pl.'s Themistokles für quellenkritische Übungen kommentiert und herausgegeben von Adolf Bauer. Leipzig: Teubner 1884.

- de puerorum educatione libellus illustratus studio Christiani Junckeri. Leipzig: Weidmann 1730.

- Agis et Cleomenes recensuit annotationem criticam prolegomena et commentarios adiecit Georg Frid. Schoemann. Greifswald: Moritz 1839.

- Ausgewählte Biographien. Für den Schulgebrauch erklärt von Otto Seifert und Friedrich Blass.

1. Bdchen.: Philopömen und Titus Quinctius Flamininus. Leipzig: Teubner 1876.

2. Bdchen.: Themistokles und Perikles. Leipig: Teubner 1872.

3. Bdchen.: Aristides und Cato. Leipzig: Teubner 1872.

Vitae parallelae Agidis et Cleomenis, Gracchorum recognovit Carolus Sintenis. Leipzig: Teubner 1867.

De musica edidit Ricardus Volkmann. Leipzig: Teubner 1856.

De puerorum educatione libellus illustratus a Petro Westhusio. Hamburg: Liebezeit 1694.

De puerorum educatione libellus illustratus a Petro Westhusio. Hamburg: Liebezeit 1702. 
Vergleichende Lebensbeschreibungen übersetzt von J. G. Klaiber. 41 Bdchen (6 Bde). Stuttgart: Metzler $1828-59$.

Symbolae criticae et palaeographicae in Pl. vitas parallelas et moralia scripsit Gregorius N. Bernardakis. Leipzig: Teubner 1879 .

Leben, Schriften und Philosophie des Plutarch von Chäronea von Richard Volkmann. Berlin: Calvary \& Co. 1869.

Lexicon Plutarcheum et vitas et opera omnia complectens composuit Daniel Wyttenbach. 2 Vol. Leipzig: Kühn (T. O. Weigel) 1843.

Polemo, Polemonis Periegetae fragmenta collegit, digessit, notis auxit L. Preller. Leipzig: Engelmann 1838.

Polyaenus, Strategematon libri octo ex recensione Eduardi Woelftlin ed. Jo. Melber. Leipzig: Teubner 1887.

Polybius, Historiae. Editionem a Lud. Dindorfio curatam retractavit Theodorus Büttner-Wobst. $4 \mathrm{Vol}$. (Vol. IV ed. L. Dindorfius.) Leipzig: Teubner 1882. 1889.1893. 1868.

Posidonius, Pos. Rhodii reliquiae doctrinae collegit atque illustravit Janus Bake. Accedit D. Wyttenbachii annotatio. Leyden: Haak \& Co. 1810.

Rhetores Graeci ex codicibus Florentinis . . emendatiores et auctiores edidit... indices adiecit Christianus Walz. 9 Vol. (10 Teile.) Stuttgart und Tübingen: Cotta 1832-36.

Scenici, Poetarum scenicorum Graecorum Aeschyli Sophoclis Euripides et Aristophanis fabulae superstites et perditarum fragmenta ex recensione et cum prolegomenis Guilelmi Dindorfii. Leipzig: Teubner 1869.

Sibyllina, Oracula cur. C. Alexandre. 2 Vol. Paris: Didot $1841-56$.

Die Sibyllinischen Weissagungen vollständig gesammelt herausgegeben von J. H. Friedländer. Leipzig: Weigel 1852.

- beigebunden: De oraculis Sibyllinis dissertatio supplementum editionis a Friedliebio exhibitae scripsit Ricardus Volkmann. Leipzig: Weigel 1853. 
Sillographi, Sillographorum Graecorum reliquiae recognovit et enarravit Curtius Wachsmuth. Leipzig: Teubner 1885.

Smyrnaens, Quintus Posthomericorum libri XIV recognovit Albertus Ziminermann. Leipzig: Teubner 1891.

Sophocles, tragoediae. Ad optimorum librorum fidem recensuit et brevibus notis instruxit C. G. A. Erfurdt. 7 Vol. Leipzig: Fleischer $1830-41$.

- tragoediae recognovit ac brevi annotatione scholarum in usum instruxit Fridericus Nevius. Leipzig: Vogel 1831.

- erklärt von F. W. Schneidewin

1. Bdchen.: Aias. 6. Aufl. von A. Nauck. Berlin: Weidmann 1871.

2. Bdchen.: Oedipus tyrannus. 6. Aufl. von A. Nauck. Berlin: Weidmann 1872.

3. Bdchen.: Oedipus auf Kolonos. 5. Aufl. von A. Nauck. Berlin: Weidmann 1870.

4. Bdchen.: Antigone. 6. Aufl. von A. Nauck. Berlin: Weidmann 1869.

5. Bdchen.: Electra. 6. Aufl. von A. Nauck. Berlin: Weidmann 1873.

6. Bdchen.: Trachinierinnen. 4. Aufl. von A. Nauck. Berlin: Weidmann 1873.

7. Bdchen.: Philoctetes. 6. Aufl. von A. Nauck. Berlin: Weidmann 1872.

- erklärt von F. W. Schneidewin

1. Bdchen.: Aias. 7. Aufl. von A. Nauck. Berlin: Weidmann 1877.

2. Bdchen: Oedipus tyrannus. 6. Aufl. von A. Nauck. Berlin: Weidmanu 1872.

3. Bdchen.: Oedipus aut Kolonos. 8. Aufl. von A. Nauck. Berlin: Weidmann 1883.

4. Bdchen.: Antigone. 9. Aufl. von A. Nauck. Berlin: Weidmann 1886.

5. Bdchen.: Elktra. 6. Aufl. von A. Nauck. Berlin: Weidmann 1873.

5. Bdchen.: Electra. 7. Aufl. von A. Nauck. Berlin: Weidmann 1877.

6. Bdchen.: Trachinierinnen. 4. Aufl. von A. Nauck. Berlin: Weidmann 1873. 
7. Bdchen: Philoctetes. 8. Aufl. von A. Nauck. Berlin: Weidmann 1882.

- tragoediae recensuit et explanavit Eduardus Wunderus. 2 Vol. Gotha und Erfurt: Hennings 1847-56.

Des Sophocles Antigone, griechisch und deutsch herausgegeben von August Boeckh. Leipzig: Teubner 1884.

- Oedipus Coloneus e recensione Petri Elmsley. Accedit Brunckii et aliorum annotatio selecta, cui et suam addidit editor. Leipzig: Hartmann 1824.

Oedipus rex. Ad optimorum librorum fidem iterum recensuit . . . C. G. A. Erfurdt. Leipzig: Fleischer 1823.

- Aiax commentario perpetuo illustravit Christ. Augustus Lobeck. Berlin: Weidmann 1866.

- König Oedipus. Nach der ältesten Handschrift .... erklärt von Franz Ritter. Leipzig: Teubner 1870.

- Antigone. Für den Schulgebrauch erklärt von Gustav Wolft. Leipzig: Teubner 1873.

- Deutsch in den Versmassen der Urschrift von J. J. C. Donner. 2 Bde. Leipzig und Heidelberg: Winter 1868.

- Lexicon Sophocleum composuit Fridericus Ellendt. 2 Vol. Königsberg: Bornträger 1835.

- Lexicon Sophocleum composuit Fridericus Ellendt. editio altera emendata curavit Hermannus Genthe. 1 Vol. Berlin: Bornträger 1872.

- Ad Sophoclis tragoedias annotationes Gulielmi Dindorfii. Oxford: Akad. Buchhdlg. 1836.

- Studien zu Sophocles von Otto Hense. Leipzig: Teubner 1880.

- Das Wesen der antiken Tragödie in ästhetischen Vorlesungen durchgeführt an beiden Oedipus des Sophocles im allgemeinen und an der Antigone insbesondere von H. Fr. W. Hinrichs. Halle: Ruff 1827.

- Ästhetischer Kommentar zu den Tragödien des Sophocles von Adolf Müller. Paderborn: Schöningh 1904.

- Beiträge zum Verständnis der Trachinierinnen des Sophocles von J. J. Öri. Berlin: Weidmann 1882.

- Commentarii in Sophoclis Oedipum Coloneum criticis commentationibus addita enarratione integri scripsit C. Reisig. Jena: Kröger 1823. 
- Scholia in Sophoclis tragoedias septem e codice ms Laurentiano descripsit Petrus Elmsley. 2 Vol. (Vol. II ed. G. Dindorfius.) Oxford: Clarendon (akad.) $1825 / 52$.

- Scholia in Sophoclis tragoedias vetera e codice Laurentiano denuo collato ed.PetrusN.Papageorgius. Leipzig:Teubner1888.

Strabo, Geographica recognovit Augustus Meineke. 3 Vol. Leipzig: Teubner 1895-98.

Suidas, Lexicon graece et latine. Ad fidem optimorum librorum exactum post Thomam Gaisfordum recensuit et annotatione critica instruxit Godofredus Bernhardy. 2 Vol. (4 Bde.) Halle und Braunschweig: Schwetschke 1852/53.

- Untersuchungen über Eudokia und Suidas von Hans Flach. Leipzig: Teubner 1879.

$\Sigma v \nu \alpha \gamma \omega \gamma \dot{\eta} \tau \varepsilon \chi \nu \tilde{\omega} \nu$, sive artium scriptores ab initiis usque ad editos Aristotelis de rhetorica libros composuit Leonhardus Spengel. Stuttgart: Cotta 1828.

Synesius, opera quae exstant omnia editore et interprete Dionysio Petavio. Accedunt Theodori Mopsuesteni scripta quae supersunt accurante et recognoscente J. P. Migne. Paris: Migne 1859.

Synesius von Cyrene. Eine biographische Charakteristik aus den letzten Zeiten des untergehenden Hellenismus von Richard Volkmann. Berlin: Ebeling \& Plahn 1869.

Themistius, orationes ex codice Mediolanensi emendatae a Guilielmo Dindorfio. Leipzig: Knobloch 1832.

Theocritus, idyllia iterum edidit et commentariis criticis atque exegeticis instruxit Ad. Th. Arm. Fritzsche. 2 Vol. $(1 \mathrm{Bd}$.) Leipzig: Pernitzsch 1868/69.

- Theocrits Gedichte erklärt von Hermann Fritzsche. 3. Aufl. besorgt von Eduard Hiller. Leipzig: Teubner 1881.

- Bion Moschus tertium edidit Augustus Meineke. Berlin: Reimer 1856.

- Lexicon Theocriteum composuit Joannes Rumpel, Leipzig: Teubner 1879.

Theognis, sententiae elegiacae, cum interpretatione et scholiis Eliae Vineti. Accesserunt et horum poetarum opera sententiosa: Phocylidis, Pythagorae, Solonis, Tyrtaei, Naumachii, Callimachi, Mimnermi, Eueni, Rhiani, Eratosthenis, Panyasidis, Lini, Menecratis, Posidippi, Metrodori, Simonidis. 
Omnia in usum scholarum collecta et ad verbum conversa per Jacobum Hertelium. Leipzig: Lamberg 1591.

- Theognis quid de rebus divinis et ethicis senserit. Dissert. inaug. phil., quam scripsit Hugo Bernhardt. Breslau: Korn 1875 .

Theophrastus, Characteres edidit Eugenius Petersen. Leipzig: Breitkopf \& Härtel 1859.

Theophylactus, Th. Simocattae historiae edidit Carolus de Boor. Leipzig: Teubner 1887.

Thomas Magister, Onomaton atticon eclogae ed. Jo.Steph.Bernard. Leyden: van der Eyk \& von Pecker 1757.

Thucydides, de bello Peloponnesiaco libri octo. ed. Ern. Friedr. Poppo. 4 Vol. 11 Teile. Leipzig: Fleischer 1821-40.

- erklärt von J. Classen. 1. 4.-8. Bdchen. 2. Aufl. Berlin: Weidmann $1873-85$.

- erklärt von J. Classen. 1. und 2. Bdchen. (1 Bd.) Berlin: Weidmann 1862/63.

- Historia belli Peloponnesiaci edidit Joannes Matthias Stahl. 2 Vol. (1 Bd.) Leipzig: Tauchnitz 1873/74.

- De bello Peloponnesiaco libri VIII. Ex interpretatione Laurentii Vallae ab Henrico Stephano iterum recognita. Frankfurt: Wechel 1589.

- Historiae recensuit Carolus Hude. Vol. I. Libri I-IV. Edit. minor. Leipzig: Teubner 1903.

- Commentar zu Thucydides' Reden, zum Gebrauch der Schüler angefertigt von Dr. L. Tillmanns. 1. Heft: Reden in Buch 1 und 2. Leipzig: Teubner 1876.

- Quaestiones grammaticae ad Thucydidem pertinentes. Auctas et correctas iterum edidit Joannes Matthias Stahl. Leipzig: Teubner 1886.

Tragicorum Graecorum fragmenta recensuit Augustus Nauck. Leipzig: Teubner 1856.

De Graecorum fabulis ad Thraces pertinentibus quaestiones criticae scripsit Fridericus Hiller de Gaertringen. Berlin: Haude und Spener 1886.

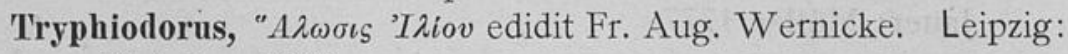
Fleischer 1819.

Tzetzes, Joannes, Antehomerica, Homerica et Posthomerica ex recensione Immanuelis Beckeri. Berlin: Reimer 1816. 
Xenophon, Opera omnia recensuit et commentariis in usum scholarum instruxit M. Frid. Aug. Bornemann. IV Vol. (Vol. II und III ed. R. Kühner, Vol. IV ed. L. Breitenbach.) Gotha und Erfurt: Hennings 1828-52.

- Opera edidit Gustavus Sauppe. V Vol. (4 Bde.) Leipzig: Tauchnitz 1865/66.

- Anabasis recognovit et cum apparatu critico edidit Ludovicus Breitenbach. Halle: Waisenhaus 1867.

- expeditio Cyri in usum scholarum emendavit C. G. Cobet. Leyden: Brill 1859.

- Anabasis Cyri recognovit et illustravit C. G. Krüger. Halle: Hemmerde \& Schwetschke 1826.

- Anabasis. Für den Schulgebrauch erklärt von Ferdinand Vollbrecht. 2 Bde. Leipzig: Teubner 1870/75.

- Cyropädie erklärt von F. K. Hertlein. 2 Bdchen. (1 Bd.) Berlin: Weidmann 1859/60.

- Institutio Cyri recensuit Arnoldus Hug. 2 Exemplare. Leipzig: Teubner 1905.

- Cyri disciplina edidit Ern. Poppo. Leipzig: Schwickert 1821.

- Hellenica. Erklärt von Ludwig Breitenbach. 3 Bdchen. Berlin: Weidmann 1873-76.

- Historia Graeca in usum scholarum emendavit C. G. Cobet. Amsterdam: Müller 1862.

- Historia Graeca recensuit Otto Keller. 2 Exemplare. Leipzig: Teubner 1898/1901.

- Hiero recensuit et interpretatus est C. H. Frotscher. Leipzig: Hartmann 1822.

- Memorabiliam Socratis dictorum libri IV recensuit Joannes August. Ernesti. 2 Exemplare. Leipzig: Fritsch 1763.

- Commentarii recognovit Walther Gilbert. Leipzig: Teubner 1898.

- Memorabilien. Mit Einleitungen und Anmerkungen herausgegeben von Moritz Seyffert. Leipzig: Holtze 1857.

- Denkwürdigkeiten desSokrateszurBeförderung der sokratischen Lehrart aus dem Griechischen übersetzt von J. C. Küntzel. Jauer: Müller 1777.

- Opuscula politica equestria venatica cum Arriani libello de venatione recensuit et interpretatus est G. A. Sauppe. Leipzig: Hahn 1838. 
Xenophon, Scripta minora recognovit Ludovicus Dindorfius. 8 Exemplare. 16 Bdchen. Leipzig: Teubner 1900/05.

- liber de vectigalibus cum commentario ed. J. C. van Deventer. Utrecht: Dannenfelser 1851.

- Prosopographia Xenophontea, illustrandis nominibus eorum, qui memorantur in Memorabilibus Socratis, Symposio et Oeconomico curavit C. G. Cobet. Leyden: 1836.

- Was ergibt sich aus dem Sprachgebrauch Xenophons in der Anabasis für die Behandlung der griechischen Syntax in der Schule? Ein Beitrag zur Methodik des griechischen Unterrichts von Artur Joost. Berlin: Weidmann 1892.

- Lexicon Xenophonteum ed. Fr. W. Sturz. IV Vol. Leipzig: Gleditsch $1801-04$.

Zosimus, Zosimi comitis et exadvocati fisci historia nova edidit Ludovicus Mendelssohn. Leipzig: Teubner 1887. 
Xenophon, Scripta

8 Exemplare.

- liber de vectigalit Utrecht: Dann

- Prosopographia X qui memorant Oeconomico cl

- Was ergibt sich der Anabasis f in der Schule? Unterrichts vol

- Lexicon Xenopho Gleditsch 1801

Zosimus, Zosimi cor Ludovicus Mer

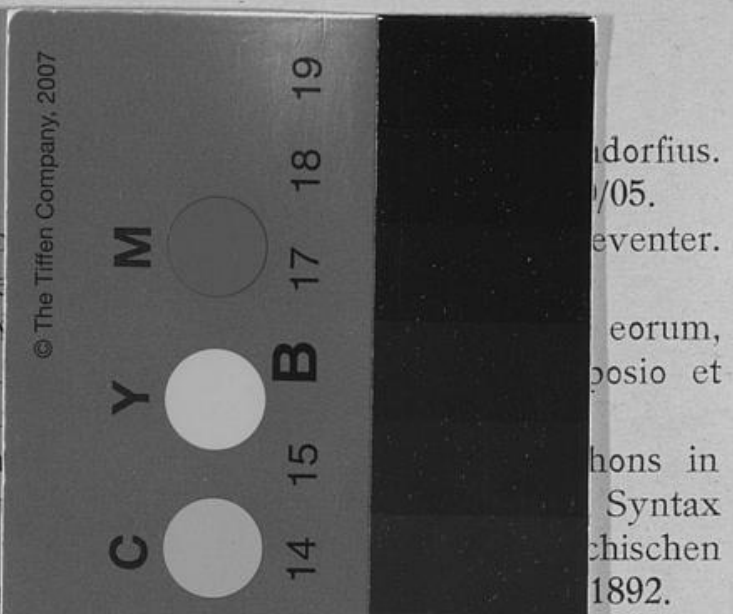

$m$

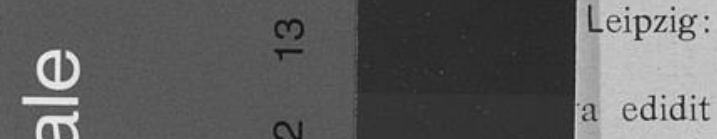




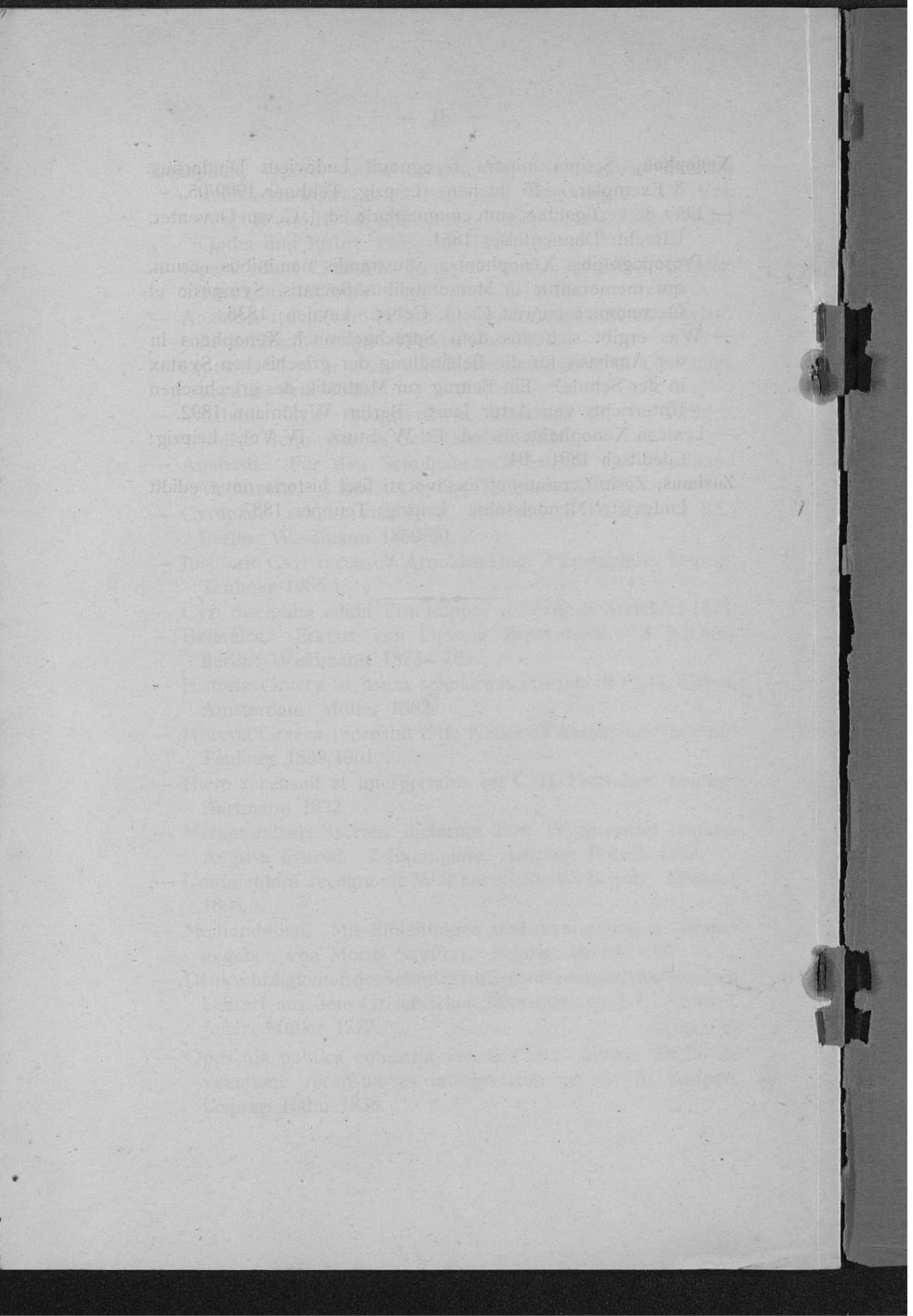




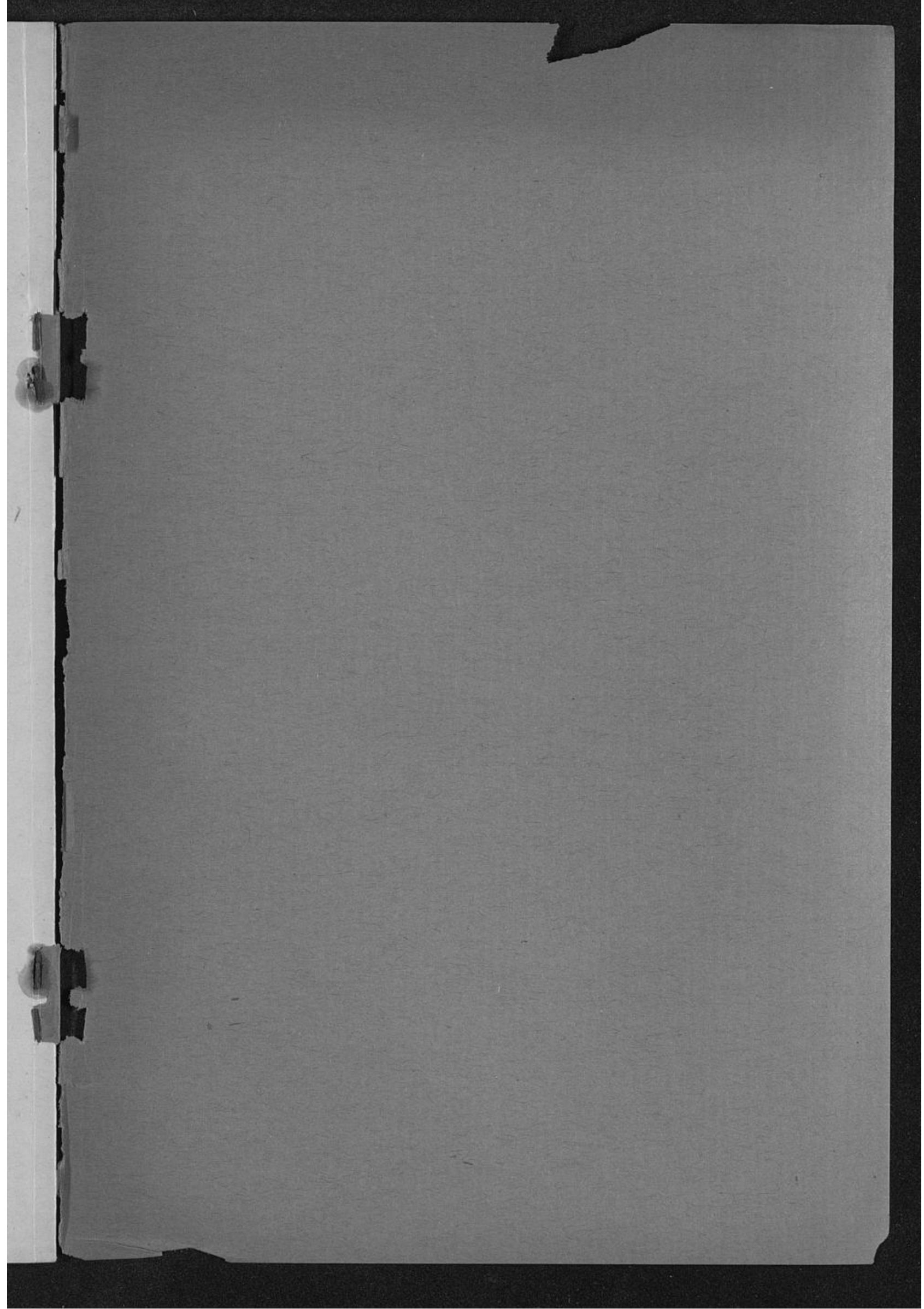

(C) Universitäts- und

Landesbibliothek Düsseldorf 
